\title{
Fatores Associados ao Consumo de Guloseimas entre Adolescentes Atendidos em um Ambulatório de Nutrição
}

\section{Factors Associated with Sweet Consumption Among Adolescents Attended in a Nutrition Ambulatory}

\author{
Andressa Pestana Brito ${ }^{1}$ \\ Adriana Sousa Rêgo ${ }^{2}$ \\ Rayane Palhano Paiva Diniz Sousa ${ }^{3}$ \\ Ana Luísa Neris Reis ${ }^{4}$ \\ Amanda Gonçalves Mota ${ }^{5}$ \\ Allanne Pereira Araújo ${ }^{6}$ \\ Flor de Maria Araújo Mendonça Silva ${ }^{7}$ \\ Janaina Maiana Abreu Barbosa ${ }^{8}$
}

\section{RESUMO}

Objetivo: Verificar os fatores associados ao consumo de guloseimas entre adolescentes atendidos em um ambulatório de nutrição. Metodologia: Estudo transversal analítico, com 217 prontuários de adolescentes, os quais foram investigadas as variáveis socioeconômicas, demográficas, estilo de vida e antropométricas. Realizou-se análise multivariada pelo método regressão de Poisson robusto por meio do programa Stata $\AA$ versão 14.0. Resultados: Dos adolescentes pesquisados, $61,8 \%$ eram do sexo feminino e $58,1 \%$ tinham idade entre 10 a 14 anos. Quanto à escolaridade dos adolescentes, $58,3 \%$ estavam cursando o ensino fundamental e $50 \%$ possuiam renda familiar até um salário-mínimo. Sobre o consumo de guloseimas, $77,1 \%$ relataram consumi-las. Com relação ao estado nutricional, $51,6 \%$ estavam com excesso de peso e $66,4 \%$ apresentaram risco cardiovascular de acordo com a Rcest. Observou-se que possuir o hábito de comer em frente à TV $(R P=1,38 ; I C=1,13-1,67)$ e ter o diagnóstico de desnutrição $(R P=0,57 ; \mathrm{IC}=0,36-0,87)$ foram associados ao consumo de guloseimas. Conclusão: Detectou-se elevado consumo de guloseimas entre os adolescentes, estando associado à desnutrição e alimentar-se em frente à TV. Espera-se que este estudo chame atenção para a importância de uma alimentação balanceada e saudável, tanto para os pais quanto para os adolescentes e, assim, sensibilizá-los quanto à prevenção de diversas patologias.

DESCRITORES

Consumo de Guloseimas. Comportamento do Adolescente. Obesidade.

\begin{abstract}
Objective: To verify the factors associated with the consumption of sweets among adolescents treated at a nutrition clinic. Methodology: A retrospective and analytical study was conducted, with 217 records of adolescents, in which socioeconomic and demographic, lifestyle and anthropometric variables were investigated. Multivariate analysis was performed using the Poisson Robust regression method, using the Stata $\circledast$ version 13.0 program. Results: Of the adolescents surveyed, $61.8 \%$ were female and $58.1 \%$ were aged 10 to 14 years. As for the education of adolescents, $58.3 \%$ were in elementary school and $50 \%$ had family income up to one minimum wage. Regarding the consumption of fats $77.1 \%$ reported consuming them. Regarding nutritional status, $51.6 \%$ were overweight and $66.4 \%$ had cardiovascular risk according to Rcest It was observed that having a habit of eating in front of the TV $(R P=1.38$; $\mathrm{Cl}=1.13-1.67)$ and having a diagnosis of malnutrition $(\mathrm{RP}=0.57 ; \mathrm{Cl}=$ $0.36-0.87)$ were associated with the consumption of sweets. Conclusion: High consumption of sweets was detected among adolescents and is associated with malnutrition and eating in front of the TV. It is expected that the present study draws attention to the importance of a balanced and healthy diet for both parents and adolescents, and thus raise their awareness of the prevention of various pathologies.
\end{abstract}

DESCRIPTORS

Candy consumption. Adolescent Behavior. Obesity.

${ }^{1}$ Nutricionista graduada pela Universidade CEUMA, São Luís, Maranhão, Brasil.

${ }^{2}$ Fisioterapeuta, Doutora em Saúde Coletiva, Universidade Federal do Maranhão - UFMA. Professora convidada do Mestrado em Gestão de Programas e Serviços de Saúde da Universidade CEUMA, São Luís, Maranhão, Brasil.

${ }^{3}$ Nutricionista graduada na Faculdade Santa Teresinha - CEST, São Luís, Maranhão, Brasil.

${ }^{4}$ Nutricionista graduada na Faculdade Santa Teresinha - CEST, Especialista em Nutrição Clínica - Faculdade Santa Teresinha, São Luís, Maranhão, Brasil.

${ }^{5}$ Nutricionista graduada na Faculdade Santa Teresinha - CEST, Especialista em Nutrição Clínica Funcional e Fitoterapia - Faculdade LABORO, São Luís, Maranhão, Brasil.

${ }^{6}$ Nutricionista, Mestra em Saúde Coletiva pela Universidade Federal do Maranhão/UFMA, docente da Faculdade Santa Terezinha - CEST, São Luís, Maranhão, Brasil.

${ }^{7}$ Psicóloga. Doutora em Saúde Coletiva pela Universidade Federal do Maranhão - UFMA. Professora Permanente do Mestrado em Gestão de Programas e Serviços de Saúde da Universidade CEUMA, São Luís, Maranhão, Brasil.

${ }^{8}$ Nutricionista. Mestre em Saúde Coletiva pela Universidade Federal do Maranhão - UFMA, docente da Faculdade Santa Terezinha CEST e da Universidade CEUMA, São Luís, Maranhão, Brasil. 
$\mathrm{A}$ Organização Mundial de Saúde (OMS) caracteriza a adolescência como a faixa etária entre 10 e 19 anos $^{1}$. Alterações metabólicas podem ocorrer nessa fase e estão associadas com um comportamento alimentar inadequado e ao sedentarismo ${ }^{2}$, contudo estes são fatores de riscos modificáveis.

A alimentação dos adolescentes tem sido caracterizada pelo elevado consumo de alimentos ultraprocessados (ricos em gorduras, açúcares e sódio) e ainda pelo consumo insuficiente de alimentos in natura, a exemplo das frutas e hortaliças ${ }^{3-5}$. Este comportamento alimentar é preocupante, visto que pode levar ao excesso de peso e a maior probabilidade de doenças crônicas não transmissíveis (DCNTs), como o câncer, o diabetes e as doenças cardiovasculares ${ }^{6}$.

Durante a pesquisa do estudo Vigilância e Prevenção na Infância e Adolescência de Doenças da Vida Adulta Não Transmissíveis - CASPIAN - IV (2011-2012) ${ }^{7}$ foi verificado que o risco de obesidade geral e de obesidade abdominal entre os participantes que raramente consumiam doces e bebidas açucaradas era menor do que naqueles que os consumiam diariamente.

Por outro lado, o comportamento alimentar dessa faixa etária está fortemente vinculado ao poder aquisitivo da família, visto que o nível socioeconômico influencia diretamente na disponibilidade, na quantidade e na qualidade dos alimentos consumidos ${ }^{8}$. Outro fator importante é a escolaridade do chefe da família que pode influenciar na escolha dos alimentos, por apresentar associação com dimensões de conhecimento em saúde e acesso à informação. Portanto, acredita-se que quanto mais elevada for a escolaridade maior a possibilidade de realizar a escolha de alimentos de melhor qualidade ${ }^{9}$.

Além desses fatores, a mídia com o seu poder de persuasão, por meio de propagandas tentadoras, pode conduzir a hábitos não desejáveis, uma vez que as propagandas se referem mais a produtos muito calóricos e pouco nutritivos ${ }^{10}$, como também o tempo de exposição excessivo em frente à TV, eleva, proporcionalmente, a um maior consumo. Diante dessas duas situações observa-se o surgimento dos maus hábitos alimentares, contribuído para um ambiente obesogênico ${ }^{11}$.

A adolescência representa uma fase importante em termos de promoção de saúde e de prevenção de fatores de risco, pois os hábitos alimentares adquiridos nesta fase, bem como na infância, tendem a perdurar ao longo da vida. Diante de evidências que associam a dieta nessa fase da vida com os riscos de doenças crônicas em adultos, este estudo teve como objetivo verificar os fatores associados ao consumo de guloseimas entre adolescentes atendidos em um ambulatório de nutrição.

\section{METODOLOGIA}

Tratou-se de um estudo transversal analítico, realizado em um ambulatório de nutrição de uma Clínica Escola vinculada à Associação dos Pais e Amigos dos Excepcionais (APAE), localizada em São Luís - MA.

A coleta dos dados foi realizada por meio de todos os prontuários dos adolescentes entre 10 a 19 anos, atendidos no setor da nutrição, o qual é referência em atendimento de adolescentes. Portanto, foram verificados 
os prontuários de adolescentes, de ambos os sexos, atendidos no período de janeiro 2010 a junho de 2015, período em que os adolescentes foram acompanhados e receberam orientação nutricional.

Foram excluídos prontuários de adolescentes com algum tipo de limitação física $(n=10)$ que inviabilizasse aferição de medidas antropométricas, prontuários de pacientes portadores de algum tipo de deficiência mental $(n=8)$, aqueles cujo item do consumo de guloseimas não estava preenchido $(n=1)$, assim como os prontuários de gestantes adolescentes $(n=1)$. Destes, foram excluídos 20 prontuários, totalizando uma amostra de 217 prontuários.

Utilizou-se as fichas de anamnese do adolescente, protocolo utilizado pelos profissionais que atendem no setor da Nutrição e foram analisados os seguintes dados: sociodemográficos e econômicos (sexo, idade, renda familiar, escolaridade dos pais e do adolescente), estilo de vida (quantidade de refeições realizadas por dia, hábito de comer em frente à TV, consumo de guloseimas e prática de atividade física) e antropométricos (peso, altura e circuferência da cintura).

Os adolescentes foram questionados quanto ao consumo de guloseimas referente à última semana e este foi categorizado em não consome e consome. O tipo de guloseimas foi dividido em: bolo de chocolate, biscoito recheado, balas, chocolates e sorvetes, refrigerantes, sucos industrializados e achocolatados e salgadinhos tipo chips, salgados fritos e batata frita.

Para a classificação do estado nutricional dos adolescentes foi utilizado o indicador IMC/l em percentis, de acordo com o sexo e foi categorizado em desnutrição (< p3), eutrofia (p3 - p85), sobrepeso (p85-p97) e obesidade $(>p 97)^{12}$. Os pontos de cortes adotados para a classificação da CC foram os propostos por Taylor et al. ${ }^{13}$ que identificam obesidade abdominal quando a $C C \geq$ percentil 80 , ajustado para a idade e o sexo.

A razão cintura/estatura (Rcest) considera a proporção de gordura central pela estatura do indivíduo, sendo um bom preditor de doença cardiovascular (DCV). O ponto de corte utilizado para classificar o risco cardiovascular nos adolescentes foram valores iguais ou superiores a $0,50^{14}$. A CC e a Rcest foram categorizadas em sem risco para DCV e com risco para DCV.

A análise dos dados foi efetuada no software Stata $®$, versão 14.0. Na análise descritiva, foram estimadas as frequências absolutas e relativas. A influência das variáveis independentes (idade, hábito de comer em frente à TV e IMC) sobre a variavel dependente (consumo de guloseimas) foi estimada e a medida de risco utilizada foi a razão de prevalência (RP), utilizando o modelo de regressão de Poisson com variância robusta. Este modelo é uma ferramenta estatística que avalia a relação entre os fatores quantitativos e uma variável resposta binária ${ }^{15}$.

Inicialmente, foram realizadas análises univariáveis com cada variável independente, sendo que as variáveis que resultaram $p<$ 0,20 foram levadas para o modelo multivariável. Posteriormente, para aceitação das associações investigadas no modelo final, foi adotado o valor de $p<0,05$. O modelo multivariável final foi construído utilizando o método backward que retira as variáveis com $p>0,05$. O presente estudo foi aprovado pelo 
Comitê de Ética em Pesquisa do Hospital São Domingos, com parecer consubstanciado sob o número 973.773/2015.

\section{RESULTADOS}

Dos 217 prontuários de adolescentes analisados, $61,8 \%$ eram do sexo feminino. $\mathrm{Na}$ estratificação por idade, 58,1\% tinham idade de 10 a 14 anos. Quanto à escolaridade dos adolescentes, 58,3\% estavam cursando o ensino fundamental e $54,8 \%$ dos pais/responsáveis tinham o ensino médio incompleto ou completo. Em relação à renda familiar, 50\% possuíam a renda mensal de até um salário mínimo (Tabela 1).

O consumo de guloseimas na última semana foi relatado por $77,1 \%$ dos adolescentes. Quanto ao tipo de guloseimas, as mais consumidas foram os bolos de chocolate, os

Tabela 1. Características sociodemográficas, econômicas e estilo de vida dos adolescentes atendidos em ambulatório de nutrição de uma Clínica Escola em São Luís - MA

\begin{tabular}{|c|c|c|}
\hline Variáveis & $\mathrm{n}$ & $\%$ \\
\hline \multicolumn{3}{|l|}{ Sexo } \\
\hline Masculino & 83 & 38,2 \\
\hline Feminino & 134 & 61,8 \\
\hline \multicolumn{3}{|l|}{ Idade } \\
\hline $10-14$ anos & 126 & 58,1 \\
\hline $15-19$ anos & 91 & 41,9 \\
\hline \multicolumn{3}{|l|}{ Escolaridade do adolescente } \\
\hline Ensino fundamental & 122 & 58,3 \\
\hline Ensino médio & 87 & 41,7 \\
\hline \multicolumn{3}{|l|}{$\begin{array}{l}\text { Escolaridade dos } \\
\text { pais/responsáveis }\end{array}$} \\
\hline $\begin{array}{l}\text { Ensino fundamental } \\
\text { incompleto e completo }\end{array}$ & 52 & 31,3 \\
\hline $\begin{array}{l}\text { Ensino médio incompleto e } \\
\text { completo }\end{array}$ & 91 & 54,8 \\
\hline $\begin{array}{l}\text { Ensino superior incompleto e } \\
\text { completo }\end{array}$ & 23 & 13,9 \\
\hline \multicolumn{3}{|l|}{ Renda familiar } \\
\hline Até $1 \mathrm{SM}$ & 103 & 50,0 \\
\hline $2 \mathrm{SM}$ & 61 & 29,8 \\
\hline$\geq 3 \mathrm{SM}$ & 42 & 20,2 \\
\hline \multicolumn{3}{|l|}{ Consumo de guloseimas } \\
\hline Não & 49 & 22,9 \\
\hline Sim & 165 & 77,1 \\
\hline \multicolumn{3}{|l|}{ Tipo de guloseima } \\
\hline $\begin{array}{l}\text { Bolo de chocolate, biscoito } \\
\text { recheado, balas, chocolates e } \\
\text { sorvetes }\end{array}$ & 81 & 42,3 \\
\hline $\begin{array}{l}\text { Refrigerantes, sucos } \\
\text { industrializados e } \\
\text { achocolatados }\end{array}$ & 61 & 28,6 \\
\hline $\begin{array}{l}\text { Salgadinhos tipo chips, } \\
\text { salgados fritos e batata frita }\end{array}$ & 62 & 29,1 \\
\hline
\end{tabular}


Tabela 2. Indicadores antropométricos de adolescentes atendidos em ambulatório de nutrição de uma Clínica Escola em São Luís - MA

\begin{tabular}{lcc}
\hline \multicolumn{1}{c}{ Variáveis } & $\mathrm{n}$ & $\%$ \\
\hline IMC & 29 & \\
Desnutrição & 89 & 13,4 \\
Eutrofia & 28 & 41,0 \\
Sobrepeso & 71 & 12,9 \\
Obesidade & & 32,7 \\
CC & 74 & 55,6 \\
Sem Risco & 59 & 44,4 \\
Com risco & 73 & 33,6 \\
RCest & 144 & 66,4 \\
Sem Risco cardiovascular &
\end{tabular}

biscoitos recheados, a bala, os chocolates e os sorvetes $(42,3 \%)$ (Tabela 1$)$.

Com relação aos indicadores antropométricos, $51,6 \%$ dos adolescentes estavam com excesso de peso de acordo com IMC/I. Quanto a CC, 55,6\% não apresentavam risco cardiovascular, entretanto $66,4 \%$ apresentaram risco cardiovascular de acordo com a Rcest (Tabela 2).

Quanto à associação do consumo de guloseimas com as variáveis sociodemográficas, o estilo de vida e as antropométricas, encontrou-se associação com hábito de comer em frente à $\operatorname{TV}(p=0,001)$ e o IMC $(p=0,001)$ (Tabela 3).

$\mathrm{Na}$ análise não ajustada, apresentar idade entre 15 a 19 anos $(R P=0,90 ; I C=0,77$ 1,05), ter o hábito de comer em frente à TV $(R P=1,47 ; I C=1,20-1,80)$ e ter o diagnóstico de desnutrição $(\mathrm{RP}=0,54 ; \mathrm{IC}=0,34-0,84)$ foram associados ao consumo de guloseimas (Tabela 4).

$\mathrm{Na}$ análise ajustada, observou-se que ter hábito de comer em frente à $T V(R P=1,38$;
$\mathrm{IC}=1,13-1,67)$ e ter o diagnóstico de desnutrição $(\mathrm{RP}=0,57 ; \mathrm{IC}=0,36-0,87)$ foram associados ao consumo de guloseimas (Tabela 5).

\section{DISCUSSÃO}

O consumo de guloseimas foi elevado entre os participantes deste estudo e ainda foi associado com o hábito de comer em frente à TV e ao diagnóstico de desnutrição.

Em estudo realizado com adolescentes das quatro últimas séries do ensino fundamental de escolas públicas municipais foi verificado um elevado consumo de alimentos ultraprocessados $(61,2 \%)$, dentre estes, os mais consumidos foram as guloseimas ( $48 \%)$, os refrigerantes $(23,5 \%)$, os embutidos $(19 \%)$ e os salgadinhos de pacote $(13,07 \%)^{16}$. Resultado semelhante foi encontrado na presente investigação, no qual um elevado percentual de adolescentes afirmou consumir guloseimas. Dados da Pesquisa Nacional de Saúde do Escolar (PeNSE) mostram que a proporção do consumo de alimentos não 
Tabela 3. Associação entre consumo de guloseimas e características socioeconômicas, estilo de vida e antropométricas de adolescentes atendidos em ambulatório de nutrição de uma Clínica Escola em São Luís - MA

\begin{tabular}{|c|c|c|c|c|c|}
\hline Variáveis & $\mathrm{n}$ & $\%$ & $n$ & $\%$ & Valor de $p$ \\
\hline & & & & & \\
\hline Sexo & & & & & 0,879 \\
\hline Masculino & 19 & 23,5 & 62 & 76,5 & \\
\hline Feminino & 30 & 22,6 & 103 & 77,4 & \\
\hline Idade & & & & & 0,171 \\
\hline $10-14$ anos & 24 & 19,5 & 99 & 80,5 & \\
\hline $15-19$ anos & 25 & 27,5 & 66 & 72,5 & \\
\hline $\begin{array}{l}\text { Escolaridade dos } \\
\text { pais/responsáveis }\end{array}$ & & & & & 0,576 \\
\hline $\begin{array}{l}\text { Ensino fundamental } \\
\text { incompleto e completo }\end{array}$ & 13 & 26 & 37 & 74 & \\
\hline $\begin{array}{l}\text { Ensino médio incompleto } \\
\text { e completo }\end{array}$ & 17 & 18,7 & 74 & 81,3 & \\
\hline $\begin{array}{l}\text { Ensino superior } \\
\text { incompleto e completo }\end{array}$ & 05 & 21,7 & 08 & 78,3 & \\
\hline $\begin{array}{l}\text { Escolaridade do } \\
\text { adolescente }\end{array}$ & & & & & 0,808 \\
\hline Ensino fundamental & 27 & 22,7 & 92 & 77,3 & \\
\hline Ensino médio & 21 & 24,1 & 66 & 75,9 & \\
\hline Renda familiar & & & & & 0,231 \\
\hline 1SM & 22 & 21,6 & 80 & 78,4 & \\
\hline $2 \mathrm{SM}$ & 18 & 30,5 & 41 & 69,5 & \\
\hline$\geq 3 \mathrm{SM}$ & 07 & 16,7 & 35 & 83,3 & \\
\hline Atividade física & & & & & 0,836 \\
\hline Não & 28 & 22,1 & 99 & 77,9 & \\
\hline Sim & 20 & 23,3 & 66 & 76,7 & \\
\hline Número de refeições diárias & & & & & 0,744 \\
\hline $1-2$ & 02 & 28,6 & 05 & 71,4 & \\
\hline $3-4$ & 15 & 20,8 & 57 & 79,2 & \\
\hline $5-6$ & 32 & 23,7 & 103 & 76,3 & \\
\hline $\begin{array}{l}\text { Hábito de comer em frente a } \\
\text { TV }\end{array}$ & & & & & 0,001 \\
\hline Não & 29 & 40,9 & 42 & 59,1 & \\
\hline Sim & 18 & 12,8 & 123 & 87,2 & \\
\hline IMC & & & & & 0,001 \\
\hline Desnutrição & 16 & 57,1 & 12 & 42,9 & \\
\hline Eutrofia & 18 & 20,7 & 69 & 79,3 & \\
\hline Sobrepeso & 04 & 14,3 & 24 & 85,7 & \\
\hline Obesidade & 11 & 15,5 & 60 & 84,5 & \\
\hline CC & & & & & 0,331 \\
\hline Sem risco cardiovascular & 16 & 21,9 & 57 & 78,1 & \\
\hline Com risco cardiovascular & 09 & 15,3 & 50 & 84,7 & \\
\hline Rcest & & & & & 0,860 \\
\hline Sem risco cardiovascular & 17 & 23,6 & 55 & 76,4 & \\
\hline Com risco cardiovascular & 32 & 22,5 & 110 & 77,5 & \\
\hline
\end{tabular}

IMC: Índice de Massa Corporal. CC: Circunferência da cintura. RCest: Razão cintura-estatura. 
Tabela 4. Análise não ajustada da associação das variáveis idade, hábito de comer em frente à TV e IMC com o consumo de guloseimas de adolescentes atendidos em ambulatório de nutrição de uma Clínica Escola em São Luís - MA

\begin{tabular}{lccc}
\hline Variável & RP & IC (95\%) & p-valor \\
\hline Idade & & & \\
$10-14$ anos & 01 & - & 01 \\
$15-19$ anos & 0,90 & $(0,77-1,05)$ & 0,185 \\
Hábito de comer em frente à TV & & & \\
Não & 01 & - & 01 \\
Sim & 1,47 & $(1,20-1,80)$ & 0,001 \\
IMC & & & \\
Eutrofia & 01 & - & 01 \\
Desnutrição & 1,54 & $(0,34-0,84)$ & 0,006 \\
Sobrepeso & 1,08 & $(0,89-1,30)$ & 0,413 \\
Obesidade & 1,06 & $(0,92-1,23)$ & 0,397 \\
\hline
\end{tabular}

RP: Razão de Prevalência; IC: Intervalo de Confiança.

Na análise ajustada, observou-se que ter hábito de comer em frente à TV $(R P=1,38 ; I C=1,13-1,67)$ e ter o diagnóstico de desnutrição $(R P=0,57 ; I C=0,36-0,87)$ foram associados ao consumo de guloseimas (Tabela 5).

Tabela 5. Análise ajustada da associação das variáveis idade, hábito de comer em frente à TV e IMC com o consumo de guloseimas de adolescentes atendidos em ambulatório de nutrição de uma Clínica Escola em São Luís - MA

\begin{tabular}{lccc}
\hline Variável & RP & IC (95\%) & p-valor \\
\hline Idade & & & \\
$10-14$ anos & 01 & - & 01 \\
$15-19$ anos & 0,94 & $(0,81-1,09)$ & 0,426 \\
Hábito de comer em frente a TV & & & \\
Não & 01 & - & 01 \\
Sim & 1,38 & $(1,13-1,67)$ & 0,001 \\
IMC & & & \\
Eutrofia & 01 & - & 01 \\
Desnutrição & 0,57 & $(0,36-0,87)$ & 0,011 \\
Sobrepeso & 1,07 & $(0,90-1,26)$ & 0,432 \\
Obesidade & 1,01 & $(0,87-1,17)$ & 0,874 \\
\hline
\end{tabular}

RP: Razão de Prevalência; IC: Intervalo de Confiança.

saudáveis variou de $15,4 \%$ a $59,6 \%$, sendo o maior percentual daqueles que consumiam guloseimas (balas, bombons, chicletes, doces, chocolates ou pirulitos) ${ }^{17}$.

O baixo consumo de frutas, verduras e legumes e o alto consumo de guloseimas pode contribuir para o excesso de peso e outras patologias como as DCV, a hipertensão, o diabetes, dentre outras doenças ${ }^{18,19}$. Ressalta-se que o sobrepeso e a obesidade na adolescência podem ser fator predisponente à permanência deste quadro na idade adulta ${ }^{20}$. 
No que se refere ao IMC, na presente pesquisa mais da metade dos adolescentes estavam com excesso de peso, o que pode ser justificado pelo fato da coleta de dados ter sido realizada em uma clínica escola, local onde os adolescentes recebiam tratamento especializado para modificar hábitos de vida. Em estudo conduzido com adolescentes do ensino fundamental, observou-se excesso de peso em $30,9 \%$ e, dos 505 entrevistados, $54,1 \%$ relataram consumir doces e refrigerantes diariamente ${ }^{21}$, corroborando com os resultados do presente estudo. Em pesquisa realizada no município de Florianópolis Santa Catarina, com o objetivo de verificar a associação entre o consumo alimentar, a atividade física, os fatores socioeconômicos e o percentual de gordura corporal em escolares, foi encontrado que a gordura corporal foi associada ao consumo de alimentos de risco (achocolatados, sucos artificiais, refrigerantes, doces, salgadinhos industrializados, batatas fritas e lanches rápidos), consumidos mais que três vezes ao dia ${ }^{22}$. Entretanto, em um estudo realizado com 784 adolescentes de escolas públicas e privadas, os adolescentes estróficos apresentaram maior consumo de alimentos ultraprocessados ( $p<0,001)$, quando comparados aos que possuíam excesso de peso ${ }^{23}$. No presente estudo ter diagnóstico de sobrepeso e de obesidade não foi fator de risco para o consumo de guloseimas, porém, ser desnutrido foi fator de proteção para os adolescentes que consumiam guloseimas.

Um estudo realizado com 14.880 estudantes de 6 a 18 anos o risco de obesidade geral e de obesidade abdominal entre os indivíduos que raramente consumiam doces foi, de aproximadamente, $25 \%$ (RC: 0,75, IC 95\%:
0,65-0,87) e $19 \%$ (RC: 0,81 , IC 95\%: 0,720,92 ) a menos do que entre os indivíduos que os consumiam diariamente, respectivamente. Além disso, o risco de obesidade geral entre os estudantes que raramente consumiam bebidas açucaradas era de aproximadamente 15\% (RC: 0,85, IC de 95\%: 0,74-0,97) a menos que entre os indivíduos que os consumiam diariamente ${ }^{7}$.

Comer em frente à TV foi fator de risco ao consumo de guloseimas no presente estudo. Inchley et al. ${ }^{24}$ afirmam que o tempo em frente à televisão está associado ao consumo de alimentos calóricos, assim como ao baixo consumo de frutas e vegetais. Em um estudo realizado com alunos de 12 a 17 anos de 124 municípios brasileiros, com uma amostra representativa de 74.589 adolescentes foi observado que mais de $70 \%$ desses jovens relataram passar duas ou mais horas por dia em frente à TV, computador ou videogames e, aproximadamente, $60 \%$ referiram realizar as refeições quase sempre ou sempre em frente à televisão ${ }^{25}$.

Em uma revisão sistemática, que analisou a associação entre o hábito de assistir à TV, o consumo alimentar e a obesidade em crianças e adolescentes, os autores indicaram que o tempo de TV exerce uma influência negativa sobre as escolhas alimentares dos adolescentes, foi encontrado um maior consumo de doces e açúcares, de refrigerantes e de leite e derivados em jovens que despendiam mais de duas horas diárias em frente à TV26. Portanto, uma forma de prevenção é evitar passar muitas horas em frete a televisão e incentivar a prática de atividade física na escola e em casa.

No presente estudo uma grande parte 
dos adolescentes tinham pais que não possuíam ensino médio completo. A escolaridade dos pais/responsáveis é um fator determinante no comportamento e no consumo alimentar de seus filhos ${ }^{27}$. Dados do Estudo de riscos cardiovasculares em adolescentes (ERICA) demonstraram uma associação significativa entre a baixa escolaridade materna e os comportamentos alimentares não saudáveis ${ }^{28}$.

Os pais são os maiores influenciadores na formação do hábito alimentar e da prática de atividade física, pois crianças e adolescentes tendem a reproduzir o comportamento dos pais $^{29}$. Em investigação realizada com escolares do nono ano do ensino fundamental de escolas das 26 capitais brasileiras e do Distrito Federal foi apontado que os adolescentes filhos de mães com ensino superior completo possuíam maior chance de praticar $300 \mathrm{mi}$ nutos ou mais de atividade física total na semana, quando comparado com as mães que não possuíam instrução ou que têm o ensino fundamental incompleto ${ }^{30}$. Além disso, adultos com maior escolaridade têm maiores níveis de prática de atividade física ${ }^{31}$, particularmente no lazer, fornecendo maior apoio social para prática de atividade física dos filhos.

Destaca-se como uma limitação deste

\section{REFERÊNCIAS}

1. Caroni MM, Bastos OM. Adolescência e autonomia: conceitos, definições e desafios Rev de Pediatria.2015; 15(1):29-34.

2. Godinho AS, Gonçalves NH, Aguiar FS, Silva Junior RF, Bauman JM, Bauman CD. Principais fatores relacionados à obesidade infantil na atualidade. RENEF. 2019; 9(13):27-40.

3. Souza AM, Barufaldi LA, Abreu GA, Giannini DT, Oliveira CL, Santos MM, et al. ERICA: In - take of macro and micronutrients of Brazilian adolescents. Rev Saúde Pública 2016; 50(supl 1):1-15. estudo a amostra ter sido do tipo não probabilística, porém, foram coletados dados de todos os prontuários dos adolescentes atendidos no ambulatório de nutrição no período de janeiro de 2010 a junho de 2015. E ainda foi utilizado o teste estatístico de Poisson com variância robusta, capaz de verificar a associação entre os fatores associados ao consumo de guloseimas.

\section{CONCLUSÃO}

Observou-se elevada prevalência de adolescentes que consumiam guloseimas e a maioria estava com sobrepeso e risco para $\mathrm{DCV}$, segundo a Rcest. O hábito de fazer as refeições em frente à TV e ter o diagnóstico de desnutrição foi associado ao consumo de guloseimas.

Esse estudo indica a necessidade de programas de educação nutricional voltados tanto para os pais/responsáveis como para os adolescentes sobre a conscientização de uma alimentação adequada e saudável para a promoção da saúde, incentivando a prática de atividade física e, assim, menor probabilidade do surgimento de doenças crônicas.

4. Andrade SC, Previdelli AN, Cesar CLG, Mar - chioni DML, Fisberg RM. Trends in diet qual - ity among adolescents, adults and older adults: a population-based study. Prev Med Rep. 2016; 4:391-396.

5. Alves, Mariane de Almeida et al. Padrões alimentares de adolescentes brasileiros por regiões geográficas: análise do Estudo de Riscos Cardiovasculares em Adolescentes (ERICA). Cad Saúde Pública. 2019; 35(6)e00153818.

6. Bloch KV, Klein CH, Szklo M, Kuschnir MC, Abreu GA Barufaldi LA, et al. ERICA: prevalências de hipertensão arterial e obesidade em adolescentes brasileiros. Rev Saúde Pública. 2016; 50(supl 1):1-13. 
7. Payab M, Kelishadi ${ }^{\mathrm{R}}$, Qorbani M, Motlagh ME, Ranjbar $\mathrm{SH}$, Ardalan Get al. Associação entre o consumo de junk food e a pressão arterial alta e obesidade em crianças e adolescentes iranianos: o Estudo Caspian-IV. J Pediatr. 2015; 91(2)196-205.

8. D'avila HF, Kirsten VR. Consumo energético proveniente de alimentos ultraprocessados por adolescentes. Rev Paul Pediatr. 2017; 35(1):54-60.

9. Carmo AS, Almeida LM, Oliveira DR, Santos LC. Influence of the Bolsa Família program on nutritional status and food frequency of schoolchildren. J Pediatr. 2016; 92(4):381-7.

10. Oliveira TS, Poll FA. A influência da publicidade na alimentação de escolares e sua relação com o excesso de peso. Rev Cippus-Unilasalle. 2016; 6:30-38.

11. Ceccatto D, Spinelli RB, Zanardo VPS, Ribeiro LA. A influência da mídia no consumo alimentar infantil: uma revisão da literatura. PERSPECTIVA, Erechim. 2018; 42(157):141-149.

12. Onis M, Onyango AW, Borghi E, Siyam A, Nishida C, Siekmann J. Development of a WHO growth reference for school-aged children and adolescents. Bull World Health Organ 2007;85: 660-7.

13. Taylor RW, Jones IE, Williams SM, Goulding A. Evaluation of waist circumference, waist-to-hip ratio, and the conicity index as screening tools for high trunk fat mass, as measured by dualenergy X-ray absorptiometry, in children aged 3-19 y. Am J Clin Nutr. 2000; 72:490-95.

14. Beck CC, Lopes AS, Pitanga FJG. Indicadores antropométricos como preditores de pressão arterial elevada em adolescentes. Arq Bras Cadiol. 2011; 96(2):126-133.

15. Coutinho LMS, Scazufca M, Menezes PR. Métodos para estimar razão de prevalência em estudos de corte transversal. Rev. Saúde Pública. 2020; 42(6):992-998.

16. Santos JC, Carvalho DMA, Pinho L. Consumo de alimentos ultraprocessados por adolescentes. Rev Adolescência e Saúde. 2019; 16(2):56-63.

17. Sousa JG, Lima LR, Fernandes CRS, Santos GM. Atividade física e hábitos alimentares de adolescentes escolares: pesquisa nacional de saúde do escolar (PENSE), 2015. RBNE. 2019; 13(77):87-93

18. Silva DFO, Lyra CO, Lima SCVC. Padrões alimentares de adolescentes e associação com fatores de risco cardiovascular: uma revisão sistemática. Ciênc Saúde Coletiva. 2016; 21(4):1181-1196.

19. Silva FMA, Smith-Menezes A, Duarte MFS. Consumo de frutas e vegetais associado a outros comportamentos de risco em adolescentes no Nordeste do Brasil. Rev Paul Pediatr. 2016; 34(3):309-315.

20. Simmonds M, Llewellyn A, Owen CG, Woolacott N. Predicting adult obesity from childhood obesity: a systematic review and meta-analysis. Obes Rev. 2016; 17(2):95-107.
21. Pinto RP, NUNES AA, Mello LM. Análise dos fatores associados ao excesso de peso em escolares. Rev Paul Pediatr. 2016; 34(4):460-468.

22. D'Avila, GL, Silva DAS, Vasconcelos FAG. Associação entre consumo alimentar, atividade física, fatores socioeconômicos e percentual de gordura corporal em escolares. Ciênc Saúde Coletiva. 2016; 4:1071-1081.

23. D'Avila HF, Kirsten VR. Consumo energético proveniente de alimentos ultra processados por adolescentes. Rev Paul Pediatr 2017; 35(1):54-60.

24. Inchey J, Currie D, Jewell J, Breda J, Barnekow V. Adolescent obesity and related behaviours: Trends and inequalities in the WHO European Region 2002-2014 Observations from the Health Behaviour in School-aged Children (HBSC) WHO collaborative cross-national study.

25. Oliveira JS, Barufaldi LA, Abreu GA, Leal VS, Brunken GS, Vasconcelos SML, Santos MM et al. ERICA: uso de telas e consumo de refeições e petiscos por adolescentes brasileiros. Rev Saúde Pública 2016; 50(sulp 1):1-9.

26. Enes CC, Lucchini BG. Tempo excessivo diante da televisão e sua influência sobre o consumo alimentar de adolescentes. Rev Nutr. 2016; 29(3):391-399.

27. Assumpção D, Dias MR, Barros MB, Fisberg RM, Barros Filho AA. Calcium intake by adolescents: a population-based health survey. J Pediatr. 2016; 92:251-259.

28. Barufaldi LA, Abreu GA, Oliveira JS, Santos DF, Fujimori E, Vasconcelos SM, et al. ERICA: prevalence of healthy eating habits among Brazilian adolescents. Rev Saúde Pública. 2016; 50(Supl.1):1-9.

29. Villa JKD, Silva AR, Santos TSS, Ribeiro AQ, Pessoa MC, Sant'Ana LFR. Padrões alimentares de crianças e determinantes socioeconômicos, comportamentais e maternos. Rev Paul Pediatr. 2015; 33(3):302-309.

30. Cruz MS, Bernal RTI, Claro RM. Tendência da prática de atividade física no lazer entre adultos no Brasil (20062016). Cad Saúde Pública. 2018; 34(10):e00114817.

31. Cureau FV, Silva TLN, Bloch KV, Fujimori E, Belfort DR, Carvalho K B et al. ERICA: leisure-time physical inactivity in Brazilian adolescents. Rev Saúde Pública. 2016; 50 (sulp 1):4s.

CORRESPONDÊNCIA

Janaina Maiana Abreu Barbosa.

Avenida Antares 898, Recanto dos Vinhais CEP: 6507070.

Tel: (98) 98219-4375

E-mail: jana_mayana@hotmail.com 